\title{
The Iranian Study of Opium and Cancer (IROPICAN): Rationale, Design, and Initial Findings
}

Maryam Hadji, PhD Student ${ }^{1,2 *}$; Hamideh Rashidian, $\mathrm{PhD}^{2 *}$; Maryam Marzban, PhD ${ }^{3,4}$; Mahin Gholipour, MD, MPH, PhD Candidate; Ahmad Naghibzadeh-Tahami, PhD Candidate ${ }^{6}$; Elham Mohebbi, DVM, MPH, PhD ${ }^{2,7}$; Elmira Ebrahimi, MS'; Bayan Hosseini MS ${ }^{2,8}$; Ali Akbar Haghdoost, MD, PhD ${ }^{9,10}$; Abbas Rezaianzadeh, MD, PhD ${ }^{11}$; Afarin Rahimi-Movaghar, MD $^{12}$; Abdolvahab Moradi, MD ${ }^{5}$; Monireh Sadat Seyyedsalehi, MS²; Reza Shirkoohi, MD, PhD²; Hossein Poustchi, MD, PhD'13; Sareh Eghtesad, MSc ${ }^{13}$; Farid Najafi, PhD ${ }^{14,15}$; Roya Safari-Faramani, PhD ${ }^{16}$; Reza Alizadeh-Navaei, PhD ${ }^{17}$; Ali Reza Ansari Moghadam, PhD ${ }^{18}$; Mahdieh Bakhshi, MSc ${ }^{18}$; Azim Nejatizadeh, MD, PhD $^{19}$; Masumeh Mahmudi, MSc ${ }^{19}$; Soudabeh Shahid-Sales, MD ${ }^{20}$; Saideh Ahmadi-Simab, MSc ${ }^{20}$; Omid Nabavian, MD ${ }^{21}$; Paolo Boffetta, MD, $\mathrm{PhD}^{22,23}$; Eero Pukkala, $\mathrm{PhD}^{1,24^{*}}$; Elisabete Weiderpass, MD, PhD ; Farin Kamangar, MD, PhD ${ }^{25^{*}}$; Kazem Zendehdel, $\mathrm{MD}^{2} \mathrm{PhD}^{2,26,27^{*}}$

'Health Sciences Unit, Faculty of Social Sciences, Tampere University, Tampere, Finland

${ }^{2}$ Cancer Research Center, Cancer Institute of Iran, Tehran University of Medical Sciences, Tehran, Iran

${ }^{3}$ Clinical Research Development Center, "The Persian Gulf Martyrs", Bushehr University of Medical Science, Bushehr, Iran

${ }^{4}$ Department of Public Health, School of Public Health, Bushehr University of Medical Science, Bushehr, Iran

${ }^{5}$ Golestan Research Center of Gastroenterology and Hepatology, Golestan University of Medical Sciences, Gorgan, Iran

${ }^{6}$ Neuroscience Research Center, Institute of Neuropharmacology, Kerman University of Medical Sciences, Kerman, Iran

${ }^{7}$ Pathology and Stem Cell Research Center, Kerman University of Medical Sciences, Kerman

${ }^{8}$ International Agency for Research on Cancer, Lyon, France

${ }^{9}$ Department of Biostatistics and Epidemiology, Kerman University of Medical Sciences, Kerman, Iran

${ }^{10}$ Regional Knowledge HUB for HIV/AIDS Surveillance, Research Centre for Modelling in Health, Institute for Future Studies in Health, Kerman University of Medical Sciences, Kerman, Iran

${ }^{11}$ Colorectal Research Center, Shiraz University of Medical Sciences, Shiraz, Iran

${ }^{12}$ Iranian National Center for Addiction Studies (INCAS), Tehran University of Medical Sciences, Tehran, Iran

${ }^{13}$ Liver and Pancreatobiliary Diseases Research Center, Digestive Diseases Research Institute, Tehran University of Medical Sciences, Tehran, Iran

${ }^{14}$ Research Center for Environmental Determinants of Health, Institute of Health, Kermanshah Medical Sciences University, Kermanshah, Iran ${ }^{15}$ Social Development and Health Promotion Research Center, Kermanshah University of Medical Sciences, Kermanshah, Iran

${ }^{16}$ Research Center for Environmental Determinants of Health, School of Public Health, Kermanshah Medical Sciences University, Kermanshah, Iran

${ }^{17}$ Gastrointestinal Cancer Research Center, Non-Communicable Diseases Institute, Mazandaran University of Medical Sciences, Sari, Iran ${ }^{18}$ Health Promotion Research Center, Zahedan University of Medical sciences, Zahedan, Iran

${ }^{19}$ Bandar-e-Abbas University of Medical Sciences, Bandar-e-Abbas, Iran

${ }^{20}$ Mashhahd University of Medical Sciences, Mashhad, Iran

${ }^{21}$ Department of Anesthesiology, Imam Hospital Complex, School of Medicine, Tehran University of Medical Sciences, Tehran, Iran

${ }^{22}$ Stony Brook Cancer Center, Stony Brook University, Stony Brook, NY, USA

${ }^{23}$ Department of Medical and Surgical Sciences, University of Bologna, Italy

${ }^{24}$ Finnish Cancer Registry - Institute for Statistical and Epidemiological Cancer Research, Helsinki, Finland

${ }^{25}$ Department of Biology, School of Computer, Mathematical, and Natural Sciences, Morgan State University, Baltimore, MD, USA

${ }^{26}$ Cancer Biology Research Center, Cancer Institute of Iran, Tehran University of Medical Sciences, Tehran, Iran

${ }^{27}$ Breast Disease Research Center, Cancer Institute of Iran, Tehran University of Medical Sciences, Tehran, Iran

\#Authors contributed to the manuscript equally.

*Corresponding Authors: Kazem Zendehdel, MD, PhD; Cancer Research Center, Cancer Institute of Iran, Tehran University of Medical Sciences, Tehran, I.R. Iran. Email: kzendeh@sina.tums.ac.ir;

Farin Kamangar, MD, PhD; Department of Biology, School of Computer, Mathematical, and Natural Sciences, Morgan State University, Baltimore, MD, USA, Email: farin.kamangar@morgan.edu;

Eero Pukkala, PhD; Faculty of Social Sciences, Tampere University, Tampere, Finland. Email: eero.pukkala@cancer.fi 


\begin{abstract}
Background: The International Agency for Research on Cancer (IARC) recently classified opium use as a Group 1 carcinogen. However, much remains to be studied on the relation between opium and cancer. We designed the Iranian Opium and Cancer (IROPICAN) study to further investigate the association of opium use and cancers of the head and neck, bladder, lung, and colon and rectum. In this paper, we describe the rationale, design, and some initial results of the IROPICAN Study.

Methods: The IROPICAN is a multi-center case-control study conducted in 10 provinces of Iran. The cases were all histologically confirmed and the controls were selected from hospital visitors who were free of cancer, were not family members or friends of the cancer patients, and were visiting the hospital for reasons other than their own ailment. The questionnaires included detailed questions on opium use (including age at initiation, duration, frequency, typical amount, and route), and potential confounders, such as tobacco use (e.g., cigarettes, nass and water-pipe), and dietary factors. Biological samples, including blood and saliva, were also collected.

Results: The validation and pilot phases showed reasonably good validity, with sensitivities of $70 \%$ and $69 \%$ for the cases and controls, respectively, in reporting opium use. The results also showed excellent reliability, with intra-class correlation coefficients of 0.96 for ever opium use and 0.88 (95\% Cl: 0.80, 0.92) for regular opium use. In the main phase, we recruited 3299 cancer cases (99\% response rate) and 3477 hospital visitor controls ( $89 \%$ response rate). The proportion of ever-use of opium was $40 \%$ among cases and $18 \%$ among controls.

Conclusion: The IROPICAN study will serve as a major resource in studies addressing the effect of opium on risk of cancers of the head and neck, bladder, lung, and colon and rectum.

Keywords: Bladder cancer, Colorectal cancer, Head and neck cancer, Lung cancer, Opium, Protocol

Cite this article as: Hadji M, Rashidian H, Marzban M, Gholipour M, Naghibzadeh-Tahami A, Haghdoost AA, et al. The Iranian Study of Opium and Cancer (IROPICAN): rationale, design, and initial findings . Arch Iran Med. 2021;24(3):167-176. doi: 10.34172/aim.2021.27.
\end{abstract}

Received: July 9, 2020, Accepted: December 11, 2020, ePublished: March 1, 2021

\section{Introduction}

Opium, a highly addictive substance obtained from the unripe seedpod of the poppy plant, is illicitly consumed by millions of people around the world, particularly in central Asian countries. ${ }^{1}$ Freshly taken from the poppy plant, opium contains alkaloids (e.g., morphine, codeine, and thebaine) and non-alkaloids (e.g., water, sugars, fat, and meconic acid). It is often minimally processed by heating, boiling, and drying, and variably adulterated with some chemicals (e.g., lead or chromium) before it reaches consumers. In the minimally processed form, opium may be consumed as raw opium (teriak), opium sap (shireh), or opium dross (sukhteh). ${ }^{2}$ These forms of opium may be ingested or smoked. Therefore, similar to tobacco, opium is a complex substance with many chemicals.

Case-control and cohort studies, conducted mainly within the past two decades, have provided substantial evidence that opium use could increase the risk of overall death, cardiovascular mortality, ${ }^{3}$ and cancer mortality. ${ }^{4,5}$ Opium use has been found to be associated with higher risk of cancers of the larynx, ${ }^{4-7}$ bladder, ${ }^{8-10}$ lung, ${ }^{10-12}$ pharynx, ${ }^{6,7,13,14}$ stomach, ${ }^{10,15,16}$ esophagus, ${ }^{10,17-20}$ pancreas ${ }^{10,21,22}$ and colon and rectum..$^{23,24}$ Using data from these studies, complemented by mechanistic studies, the International Agency for Research on Cancer (IARC) recently classified opium use a Group 1 carcinogen, i.e. carcinogenic in humans. ${ }^{25,26}$

The IARC Working Group concluded that opium use causes cancers of the larynx, lung, and bladder. However, there remain substantial knowledge gaps for the association of opium use and cancer. For example, elucidating the magnitude of the association for these cancers and studying the interaction of opium use with tobacco smoking for these cancers need further investigation. Despite the availability of some credible data, it is unclear whether opium causes cancers of the esophagus, stomach, or pancreas. Data for some cancers, most notably those of colon and rectum, are contradictory. ${ }^{10,24}$ While epidemiologic findings so far suggest that consumption of opium in its various forms (teriak, shireh and sukhteh) and major exposure routes (ingestion and smoking) would be carcinogenic, there is a clear need for further data to support these findings. To advance the science in this field, we initiated the Iranian Opium and Cancer (IROPICAN) Study in 2012, to evaluate the association of opium exposure with risk of cancers of the head and neck, lung, bladder, and colon and rectum.

We aimed to advance the field by planning studies that had a substantially larger sample size than any studies before, and to collect detailed data on opium use needed for dose-response analyses, controlling for potential confounders, and evaluation of possible reverse causality. ${ }^{27}$ The findings for head and neck cancer are already published which showed odds ratio (OR) $(3.76,95 \% \mathrm{CI}$ : 2.96-4.79) for regular opium use ${ }^{6}$ and contributed to the recent IARC Working Group conclusions, particularly for laryngeal and pharyngeal cancer evaluation. The data for the remaining cancer sites are being analyzed. While the IROPICAN study is primarily focused on opium use, the researchers have collected substantial data on other important exposures (e.g., waterpipe use) and collected biological samples that can be used for a host of epidemiological studies. Therefore, a clear and thorough description of methods and available data will be of interest for the readers of future studies.

The objective of this paper is to introduce the 
IROPICAN study, including the study setting, design of the questionnaires, validity and reliability of the responses, biological samples collected during the study, the rationale for selection of controls, and the potential for in-depth evaluation of cancer risk factors using the material collected in these studies.

\section{Materials and Methods}

Study Setting

The IROPICAN case-control study was conducted in Iran, a country with a high consumption rate of opium ${ }^{1}$ that has a strong infrastructure for conducting large-scale studies. It was a multi-center study, conducted in 10 provinces that were estimated to have medium to high opium use, ${ }^{28}$ namely Tehran, Kerman, Fars, Golestan, Khorasan-Razavi, Kermanshah, Mazandaran, Bushehr, Hormozgan, and Sistan-Baluchestan (Figure 1), to evaluate the association between opium consumption and risk of cancers of the head and neck, lung, bladder, and colon and rectum. The regions were selected based on prevalence of opium use and availability of cancer care centers for recruiting patients and contols. Of these, Tehran, Kerman, Fars, and Golestan were the first provices to join the study in 2014 to 2015. Later, Kemanshah, Mazandaran, and KhorasanRazavi joined in 2017 and 2018, followed by Hormozgan, Sistan-Blouchestan, and Bushehr in 2018 to 2020.

\section{Questionnaires}

We developed a detailed questionnaire and administered it to all study participants. This questionnaire was adapted from the Golestan Cohort Study, ${ }^{29}$ because it had detailed data on opium use, had been tested extensively in the field (in the cohort and several case-control studies), was the basis of many publications, and could be eventually

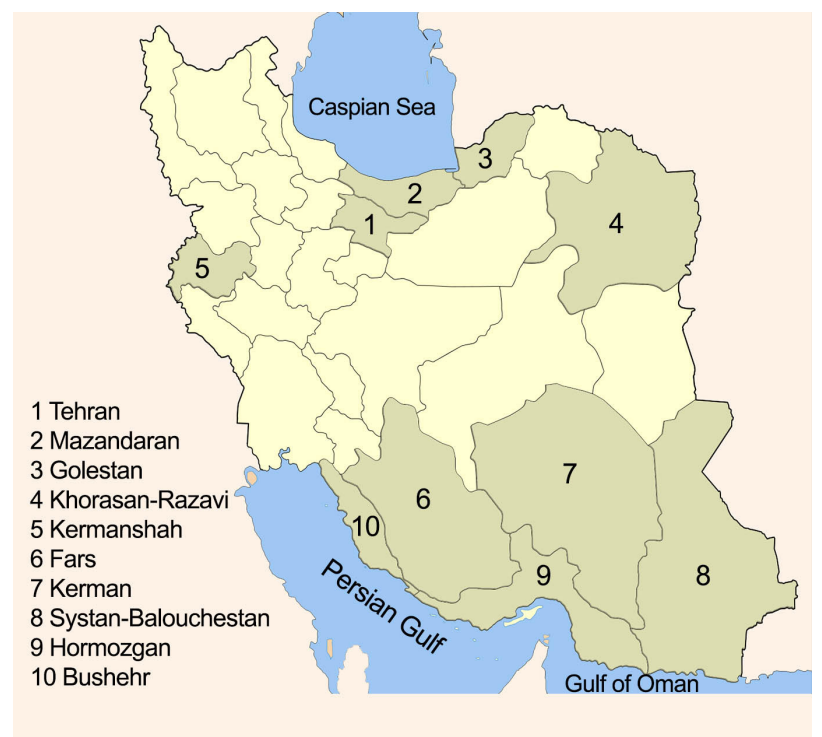

Figure 1. Distribution of the Iran Opium and Cancer (IROPICAN) study sites in different provinces of Iran. used for combined analyses of data. The IROPICAN questionnaire include detailed questions on opium use, including types of opium used (e.g. teriak, shireh, sukhteh), routes of opium use (smoking versus ingestion), age of initiating and stopping opium use, and frequency and amount of opium use. The questionnaire also include extensive data on covariates, such as: demographic and socioeconomic factors (e.g. age, ethnicity, rural/urban status, education, occupational history, various amenities owned; physical activity (using the international physical activity questionnaire, IPAQ ${ }^{30}$ ); information on various aspects of health (oral health, female reproductive history, history of various diseases, personal and family history of cancer); life-long consumption of tobacco in the forms of cigarettes, water-pipe, pipe, naswar, and chopogh (a special pipe); history of alcohol consumption; and history of opioid use (e.g. diphenoxylate, methadone, morphine, codeine); history of other illicit drug use (e.g. heroin, heroin crack, hashish, tramadol, methadone). The crack used in Iran is heroin-based and different from the cocaine-based ${ }^{34}$ crack used in Western countries. We used a validated and reproducible 130 -item food frequency questionnaire similar to that used in the Persian Cohort Study, a large study aiming to recruit 150000 Iranians aged 35-70 years from 18 regions of Iran. ${ }^{31}$

Physical Examination and Biological Sample Collection All prospective study participants underwent physical examination using a standard protocol and performed by trained personnel. Weight was measured using a portable non-digital weight scale (Fazeni), height with a height scale (Seca), and blood pressure was measured with a digital scale (Haalar). ${ }^{32,33}$

Blood samples were collected to study the presence of different opium adulterants ${ }^{34}$ and their associations with selected cancers. We collected $12 \mathrm{~mL}$ of venous whole blood for each cancer case and control. From this sample, $6 \mathrm{~mL}$ is kept in a vacutainer tube with EDTA anticoagulant for DNA extraction; $3 \mathrm{~mL}$ is stored for RNA extraction, and 3 $\mathrm{mL}$ is stored in a vacutainer tube without anticoagulant for serum extraction. One drop of blood is stored in a DNA banking card. Oral saliva from head and neck cancer cases and their controls is collected for assessing the prevalence of human papillomavirus (HPV) and its serotypes.

Blood and saliva samples were stored at minus 20 degrees Celsius in each province and shipped to the main center every month (maximum two months after collection) for storage at minus 80 degrees Celsius. DNA extraction was done using the salting-out method at the Cancer Research Center (CRC), Cancer Institute of Iran; extracted DNAs were stored at minus 20 degrees Celsius. These biological samples will be used for several other projects in the future including potential genome-wide association studies in international consortia. 
Interviewer Training and Project Management

Interviewers with a bachelor's or master's degree in public health or biology were trained intensively to use a standard protocol, to carry out standardized interviews, and to collect samples for the study. One experienced researcher in each province was responsible for the management of field-work and data collection. In addition, a central coordinator based in the main center (Cancer Research Center in Tehran University) tracked all implemented work daily, and made a visit to each center twice a year to supervisor the operations. Each center regularly reported the number of cases and controls recruited.

Choosing the Most Appropriate Control Group Using a Validation Study

Self-reported opium use in the Golesan Cohort Study was strongly correlated with urine morphine metabolites in a sample of 130 study cohort participants ${ }^{35}$; using urine codeine or morphine as the gold standard for use of opium, self-report had a sensitivity of 0.93 and a specificity of 0.89 . However, the validity of this questionnaire outside the Golestan Province was unclear. Therefore, in JuneJuly 2016, a validation study was conducted in four provinces that were first selected as study sites - Kerman, Fars, Golestan, and Tehran - to estimate the magnitude of underreporting and to determine the best potential control group.

The two potential control choices were: 1) hospitalized non-cancer patients (178 men); and 2) healthy hospital visitors who were companions of patients with chronic diseases (186 men). The prevalence of self-reported regular opioid use, defined as using at least once a week for six months, was 36\% (95\% confidence interval: $28 \%, 43 \%)$ in hospitalized patients versus 19\% (95\% CI: $13 \%, 25 \%)$ in healthy individuals. ${ }^{36}$ Compared to a gold standard of using urine rapid drug screen and thin layer chromatography, self-reported use had a sensitivity of $77 \%$ in hospitalized patients and $69 \%$ in healthy visitors $(P=0.4) .{ }^{36}$ After correction for sensitivity, the frequency of regular opioid use (primarily opium use) was $47 \%$ and $28 \%$ in hospitalized patients and healthy visitors individuals, respectively. Because $47 \%$ was a substantial overestimation of the estimated prevalence of use in the population, and that sensitivity of self-reported use was comparable in the two control groups, we opted for the healthy visitor controls in the IROPICAN Study.

\section{Validation of Self-reported Opium Use in Cases}

We studied the sensitivity of self-reported opium use in a study of 100 cancer patients (with various types of cancer) who were slated for surgery in the Cancer Institute of Tehran University of Medical Sciences. We recruited only men, to make the results comparable with the validation study in controls, with mean age of 61.5 (standard deviation, SD: 16.3) years. Most cancer patients had used opioids to alleviate pain; therefore, urine test results could not be used to validate responses. Instead, we used patient's response to the anesthesiologist's questions as gold standard; anesthesiologists routinely ask these questions before each surgery to prevent withdrawal syndrome. Sensitivity of self-reported opium use was $69.6 \%$.

\section{Reliability Study}

The Golestan Cohort Study questionnaire had shown excellent reliability for ever opium use report, with a kappa of $0.96 .{ }^{35}$ To confirm again, we assessed the reliability of the questions of drug, tobacco, and alcohol use in 57 patients ( 47 men and 10 women) who were referred to an addiction control center. We used a test-retest approach, with the second administration done two weeks after the first. The intraclass correlation coefficient (ICC) of lifetime opium use was 0.96 , and the ICC for regular use of opium was 0.88 (95\% CI, 0.80, 0.92). The ICC was 0.78 (95\% CI: $0.63,0.90)$ for method of opium use and 0.97 (95\% CI: $0.95,0.98$ ) for opium type (Table 1).

\section{Pilot Study}

In the pilot study conducted in January-April 2017, we recruited patients with four types of cancer as the case group and healthy visitors as the control group from referral cancer care centers and clinics in the four provinces

Table 1. Intra-class Correlation (ICC) and $95 \%$ Confidence Interval $(\mathrm{Cl})$ for Questions on Opium, Other Drugs, Tobacco and Alcohol Use in the Iranian Opium and Cancer (IROPICAN) Multi-center Case-Control Study, 2015-2020

\begin{tabular}{llc}
\hline Variables & ICC & $\mathbf{9 5} \% \mathbf{C I}$ \\
\hline Ever cigarette use & 0.96 & $(0.94,0.97)$ \\
\hline Regular cigarette use & 0.95 & $(0.91,0.97)$ \\
\hline Ever water pipe use & 0.88 & $(0.80,0.93)$ \\
\hline Regular water pipe use & 0.99 & $(0.98,0.99)$ \\
\hline Ever chopogh use & 0.88 & $(0.80,0.93)$ \\
\hline Regular chopogh use & 0.96 & $(0.93,0.98)$ \\
\hline Ever opium use & 0.96 & $(0.93,0.96)$ \\
\hline Regular opium use & 0.88 & $(0.80,0.92)$ \\
\hline Ever heroin use & 0.95 & $(0.92,0.97)$ \\
\hline Regular heroin use & 0.92 & $(0.86,0.95)$ \\
\hline Ever hashish use & 0.98 & $(0.96,0.98)$ \\
\hline Regular hashish use & 0.91 & $(0.85,0.95)$ \\
\hline Ever codeine use & 0.89 & $(0.81,0.93)$ \\
\hline Regular codeine use & 0.94 & $(0.89,0.96)$ \\
\hline Ever tramadol use & 0.90 & $(0.82,0.94)$ \\
\hline Regular tramadol use & 0.84 & $(0.72,0.91)$ \\
\hline Ever diphenoxylate use & 0.95 & $(0.92,0.97)$ \\
\hline Regular diphenoxylate use & 0.84 & $(0.71,0.91)$ \\
\hline Ever methadone use & 0.94 & $(0.90,0.96)$ \\
\hline Regular methadone use & 0.97 & $(0.96,0.98)$ \\
\hline Ever alcohol use & 0.98 & $(0.96,0.98)$ \\
\hline Regular alcohol use & 0.99 & $0.95,0.98)$ \\
\hline Type of opium & 0.97 & $0.78)$ \\
\hline Route of opium use & & \\
\hline & $0.99)$ \\
\hline
\end{tabular}


that were initially selected as study sites (Tehran, Shiraz, Kerman, and Golestan). The objectives of the pilot study were to: (a) determine the feasibility of the case-control study; (b) optimize the questionnaires; $(c)$ train partnering research institutions for data collection; (d) assess the geographic variation of opium use and other opiates in different regions of Iran; and (e) optimize biological sample collection methods. The pilot study showed that conducting the study was feasible. Based on pilot study findings, we made minor revisions to the questionnaire, primarily focused on questions regarding opium use and other drug use. For example, a question about frequency of opium use per day/week/month/year was added. The other aims of the pilot study were also achieved. Only minor changes were made in sample collection and methodology when the full project was set up. Partnering institutions received the required training. Also, variability of the opium consumption was determined. Following a successful pilot study, we calculated the required sample size and started recruitment of the cases and controls.

\section{Sample Size Calculation}

While it is difficult to accurately estimate the prevalence of opium use as an illicit substance ${ }^{37}$ the use of which is also associated with some social stigma, ${ }^{38}$ the results of national surveys and our pilot results suggested that a prevalence of approximately $20 \%$ among the controls was a reasonable assumption. Assuming a prevalence of $20 \%$ of opium use in the Iranian population ${ }^{39,40}$ living in high prevalence regions, $90 \%$ power and a two-sided significance level of 0.05 , we estimated that 250 cases and 250 controls would be sufficient to detect an odds ratio of 2.0. However, we chose to aim at a sample size of 800 cases for each type of cancer (i.e. 3200 cancer cases overall) and 3200 common controls, to allow for various subgroup analyses. ${ }^{39,40}$ Where possible, we exceeded this number. As of the date this report, we included 743 lung cancers, 920 colorectal cancers, 717 bladder cancers, and 919 head and neck cancers.

\section{Case Recruitment}

Patients with confirmed histological primary cancers of the lung (ICD-10: C33-34), colorectum (C18-21), bladder (C67), or squamous cell carcinoma (SCC) of the head and neck (i.e., oral cavity (C03-06), larynx (C32) and pharynx (C10-C14)) admitted to referral hospitals of the universities of medical sciences of 10 provinces (Tehran, Kerman, Fars, Golestan, Khorasan-Razavi, Kermanshah, Mazandaran, Bushehr, Hormozgan, and SistanBaluchestan), were recruited as cases. We only included newly diagnosed resident cancer cases, defined as those who had received a diagnosis within one year prior to the date of interview and who had resided at least two years in the study region. Other inclusion criteria were being of Iranian nationality, ability to speak and understand the
Farsi language, ability to participate in an interview taking about 80 minutes, and being in the age range of 30 to 75 years. Patients with metastatic cancers, second primary cancers, and those without a confirmed pathology report were excluded. Pregnant and nursing women were also excluded from the study.

\section{Control Recruitment}

The control study participants (hospital visitors) were enrolled concurrently with the cases. The controls (who had to be free of cancer) were chosen from the relatives or friends of patients from non-oncology wards, or others who visited the hospital for reasons other than receiving treatment. Choosing healthy visitors was a conclusion that we had reached during the validation studies (see above). Another rationale for this choice of controls was that we aimed at excluding those who were ill (hence, more likely than the general population to use opium) and family members or friends of patients with cancer (who are likely to share many habits, including opium use) with the cases. Other inclusion criteria were similar to those of cases, i.e., being of Iranian nationality, ability to speak and understand the Farsi (Persian) language, ability to participate in an interview taking about 80 minutes, and being in the age range of 30 to 75 years. We aimed at enrolling controls who were frequency-matched to the cases for gender, age (five year intervals), and place of residence (by province and capital city/non-capital city).

\section{Data Processing and Exposure Assessment}

Ever-use of opium was defined as using opium at least once during lifetime and regular opium use was defined as using opium at least once a week for at least six consecutive months. The cumulative amount of lifetime opium use was defined as the amount of opium use (grams per day) multiplied by days of opium use during lifetime.

We calculated pack-years, starting age and stopping age of cigarette use to be used as the main measure of exposure to cigarette smoking. Other types of tobacco used in the area are water pipe, pipe, and chopogh (a special type of pipe with a long tube).$^{41}$ For water pipe, the head-year unit was calculated for cumulative use. ${ }^{45}$ For estimation of use of alcohol, one standardized unit was defined as follows: $340 \mathrm{cc}$ of beer; $150 \mathrm{cc}$ of wine or champagne; or $40 \mathrm{cc}$ of vodka, fortified wine or whisky. We provided an album containing pictures of standard units such as pilsner, glass, cocktail and tablespoon to participants to report their portion size according to the standardized units.

We created a composite variable for socioeconomic status using a host of variables, as described elsewhere. ${ }^{42}$

\section{Results}

Overall, 3299 cancer patients (743 with lung cancer, 920 with colorectal cancer, 717 with bladder cancer and 919 with head or neck cancer) and 3477 controls participated 
in the main study. The response rate was 99\% (3299 out of 3314 ) among the cases who were capable to participate and $89 \%$ (3477 out of 3925) among the controls.

\section{Non-participants}

Only $1 \%$ of the invited cases and $11 \%$ of the invited controls chose not to participate in the study. The main stated reasons for non-participation were sickness and lethargy among cancer patients (93\% of the non-participants) and lack of time or unwillingness to donate a biological sample among controls (83\% of the non-participants). Among the 15 cases who refused to participate, the mean age was 58.8 (SD 11.2) years and 66\% were males. Among controls who refused to participate, the mean age was 58.3 (SD 10.9) years and 71\% were males.

Demographic and Geographic Characteristics of the Participants

Table 2 shows the demographic characteristics and geographic distribution of the study participants by case status. The mean age on the interview date was 59.9 (SD 11.9) years for cases and 57.3 (SD 11.6) years for controls.

\section{Opium Use}

In all, $39.8 \%$ of cases and $17.5 \%$ of controls reported ever use of opium. Patients may start using opium to alleviate their pain - a prodromal symptom of subsequently diagnosed cancer. Opium's alkaloid constituents, in addition to having narcotic effects, may be used for medicinal purposes. For example, morphine has strong analgesic effects and may be used to alleviate pain in cancer patients, or codeine may be used to suppress cough. ${ }^{43,44}$ If cases and controls who started using opium less than 3 years before their interview date were classified as nonusers, $35.1 \%$ of cases and $13.0 \%$ of controls were classified as opium users (Table 3).

The median of lifetime use of opium among users were $0.9\left(25^{\text {th }}\right.$ and $75^{\text {th }}$ percentiles 0.3 and 3.0) gram per day among cases and $0.4(0.1,2.0)$ gram per day among controls.

The types of opium consumed differed among opium users: $28.0 \%$ of cases and $11.8 \%$ of controls used teriak (Table 3). The most common opium consumption method was smoking alone (21.4\% of cases, $10.9 \%$ of controls).

\section{Tobacco Use}

The prevalence of cigarette ever use was $51.4 \%$ and $33.4 \%$ among cases and controls, respectively. The proportion of regular cigarette smokers was $48.4 \%$ in cases and $28.1 \%$ in controls._The prevalence of regular water-pipe use among cases and controls was $9.1 \%$ and $7.5 \%$, respectively. The prevalence of regularly chewing nass was 1.7 among cases and 0.8 among controls (Table 3).

\section{Biological Samples}

Biological samples including whole blood, serum, plasma,

Table 2. Demographic Characteristics of Cases and Controls Enrolled in the Multi-center Case-Control Study of Opium and Cancer (IROPICAN Study), 20152020, by Cancer Site

\begin{tabular}{|c|c|c|c|c|c|}
\hline \multirow{2}{*}{ Variable } & \multicolumn{4}{|c|}{ Cases, Site No. (\%) } & \multirow{2}{*}{ Controls, No. $(\%)$} \\
\hline & Lung & Colorectal & Bladder & Head and Neck & \\
\hline Total & $743(100)$ & $920(100)$ & $717(100)$ & $919(100)$ & $3477(100)$ \\
\hline \multicolumn{6}{|l|}{ Age } \\
\hline$<30$ & $2(0.3)$ & $7(0.8)$ & $3(0.4)$ & $9(1.0)$ & $21(0.6)$ \\
\hline 30-39 & $22(3.0)$ & $60(6.5)$ & $11(1.5)$ & $55(6.0)$ & $236(6.8)$ \\
\hline $40-49$ & $91(12.3)$ & $133(14.5)$ & $50(7.0)$ & 145 (15.8) & $559(16.1)$ \\
\hline $50-59$ & $231(31.1)$ & $258(28.0)$ & $181(25.2)$ & 277 (30.1) & $1070(30.8)$ \\
\hline $60-69$ & $257(34.6)$ & $274(29.8)$ & $267(37.2)$ & $295(32.1)$ & $1092(31.4)$ \\
\hline$>70$ & $138(18.6)$ & $187(203)$ & $205(28.6)$ & $138(15.0)$ & $499(14.4)$ \\
\hline Missing & $2(0.3)$ & $1(0.1)$ & - & - & - \\
\hline \multicolumn{6}{|l|}{ Gender } \\
\hline Female & $181(24.4)$ & $387(41.1)$ & $93(13.0)$ & $232(25.2)$ & $1077(31.0)$ \\
\hline Male & $562(75.6)$ & $533(57.9)$ & $624(87.0)$ & $687(74.8)$ & $2400(69.0)$ \\
\hline \multicolumn{6}{|l|}{ Province } \\
\hline Tehran & 148(19.9) & $170(18.5)$ & $139(19.4)$ & $163(17.7)$ & 816 (23.5) \\
\hline Fars & $233(31.4)$ & $265(28.8)$ & $166(23.2)$ & $379(41.2)$ & $943(27.1)$ \\
\hline Kerman & $128(17.2)$ & $112(12.2)$ & $150(20.9)$ & $160(17.4)$ & $525(15.1)$ \\
\hline Golestan & $64(8.6)$ & $156(17)$ & $46(6.4)$ & $46(5.0)$ & 374 (10.8) \\
\hline Mazandaran & $36(4.9)$ & $59(6.4)$ & $24(3.4)$ & $17(1.9)$ & $136(3.9)$ \\
\hline Kermanshah & $42(5.7)$ & $71(7.7)$ & $52(7.3)$ & $37(4.0)$ & $251(7.2)$ \\
\hline Khorasan-Razavi & $17(2.3)$ & $87(9.5)$ & $30(4.2)$ & $44(4.8)$ & $170(4.9)$ \\
\hline Bushehr & $38(5.1)$ & $*$ & $56(7.8)$ & $* *$ & $84(2.4)$ \\
\hline Hormozgan & $20(2.3)$ & $*$ & $27(3.8)$ & $37(4.0)$ & $78(1.9)$ \\
\hline Sistan-Balouchestan & $17(2.3)$ & * & $27(3.8)$ & $36(3.9)$ & $100(2.9)$ \\
\hline
\end{tabular}

*Colorectal cancer cases were not enrolled in Bushehr, Hormozgan, and Sistan-Baluchestan.

**Head and neck cancer were not enrolled in Bushehr. 
Table 3. Opium and Tobacco Use among the Cases and Controls in the Iranian Opium and Cancer (IROPICAN) Multi-center Case-Control Study of Opium and Cancer (IROPICAN Study), 2015-2020

\begin{tabular}{|c|c|c|}
\hline Variable & Cases, No. $(\%)$ & Controls, No. $(\%)$ \\
\hline Total & $3299(100)$ & $3477(100)$ \\
\hline \multicolumn{3}{|l|}{ Opium use* } \\
\hline Never used & $1985(60.2)$ & $2870(82.5)$ \\
\hline Ever used & $1314(39.8)$ & $607(17.5)$ \\
\hline Non-regular use & $129(3.9)$ & $139(4.0)$ \\
\hline Regular use & 1185 (35.9) & $468(13.5)$ \\
\hline \multicolumn{3}{|l|}{ Median opium amount } \\
\hline Never used & $1985(60.1)$ & $2870(82.5)$ \\
\hline Non-regular use & $129(3.9)$ & $139(0.4)$ \\
\hline$\leq 0.4 \mathrm{~g} / \mathrm{d}$ & $315(10.0)$ & $217(6.2)$ \\
\hline$>0.4 \mathrm{~g} / \mathrm{d}$ & $870(26.4)$ & $251(7.2)$ \\
\hline \multicolumn{3}{|l|}{ Opium use duration } \\
\hline Never used & $1985(60.1)$ & $2870(82.5)$ \\
\hline Non-regular use & $129(3.9)$ & $139(4.0)$ \\
\hline$\leq 17$ years & $416(12.6)$ & $243(7.0)$ \\
\hline$>17$ years & $769(23.3)$ & $225(6.5)$ \\
\hline \multicolumn{3}{|l|}{ Initiation age of opium use } \\
\hline Never used & $1985(60.1)$ & $2870(82.5)$ \\
\hline$\leq 30$ years & 648 (19.6) & $296(8.5)$ \\
\hline$>30$ years & $577(17.5)$ & $262(7.5)$ \\
\hline Unknown & $89(2.7)$ & $49(1.4)$ \\
\hline \multicolumn{3}{|l|}{ Type of opium use } \\
\hline Never used & $1985(60.1)$ & $2870(82.5)$ \\
\hline Non-regular use & $129(3.9)$ & $139(4.0)$ \\
\hline Crude opium (Teriak) & $922(28.0)$ & $411(11.8)$ \\
\hline Opium juice (Shireh) & $89(2.7)$ & $30(0.9)$ \\
\hline Opium dross (Sookhteh) & $3(0.1)$ & $2(0.06)$ \\
\hline More than one type & $171(5.2)$ & $25(0.7)$ \\
\hline \multicolumn{3}{|l|}{ Route of opium use } \\
\hline Never used & $1985(60.1)$ & $2870(82.5)$ \\
\hline Non-regular use & $129(3.9)$ & $139(4.0)$ \\
\hline Smoking & $707(21.4)$ & $380(10.9)$ \\
\hline Ingestion & $135(4.1)$ & $31(0.9)$ \\
\hline Inhalation & $21(0.6)$ & $10(0.3)$ \\
\hline More than one route & $318(9.6)$ & $45(1.3)$ \\
\hline Unknown & $4(0.1)$ & $2(0.06)$ \\
\hline \multicolumn{3}{|l|}{ Cigarette use } \\
\hline Never used & $1602(48.6)$ & $2316(66.6)$ \\
\hline Non-regular use & $101(3.1)$ & $184(5.3)$ \\
\hline Regular use & $1596(48.3)$ & $977(28.1)$ \\
\hline \multicolumn{3}{|l|}{ Tobacco use } \\
\hline Never used & $2932(88.9)$ & 3177 (91.4) \\
\hline Regular water pipe use & $300(9.1)$ & $261(7.5)$ \\
\hline Regular chopogh use & $2(0.06)$ & $1(0.03)$ \\
\hline Regular pipe use & $9(0.3)$ & $9(0.3)$ \\
\hline Regular nass & $56(1.7)$ & $29(0.8)$ \\
\hline
\end{tabular}

extracted DNA for 2441 cases and 2768 controls, and saliva sample for 657 head and neck cancer cases, and 677 controls are available.

\section{Discussion}

The IROPICAN was designed and tested in various phases to serve as a high-quality study to evaluate the causal association of opium with various cancers. The results of the validation and pilot phases guided the investigators to choose the best potential control group, and to optimize the questionnaire, data collection, and biological sample collection methods. They also showed that the main study was feasible. The response rate was high in both cases and controls. Matching of controls for age, gender, province, and place of residence was feasible. However, the matching was not perfect, as the controls were slightly younger than the cases and they were more likely to be from Tehran and Fars provinces (Figure 2), two active centers that joined the study very early on.

The IROPICAN study has several advantages in evaluating the association of opium and cancers. This study has detailed information on opium use, including age of initiation; typical amount, frequency, duration of use; and types and routes of opium use. It also has data on potential important confounders, such as age, sex, tobacco use, and socioeconomic status. Since we have detailed data on amount of use over time, we will be able to examine dose-response associations. Reverse causality can be addressed in this study by removing those who initiated opium use within the three years prior to the interview. This is important, as some people with cancer may use opium to alleviate their cancer-related pain. ${ }^{22}$ Indeed, the IROPICAN Study has already contributed to the evaluation of carcinogenicity of opium use. ${ }^{6,25}$

A major challenge in health studies on the effects of opium use is gathering reliable data on usage. ${ }^{45}$ We believe that we are using a reasonably accurate method to collect data on opium use and other banned substances. We found the sensitivity of self-reporting of opium use at $77 \%$ and $69 \%$ among disease-controls and visitor-controls, respectively. ${ }^{36}$ The high participation rates and the low denial of drug consumption in controls in our study may be due to the substantial training and supervision of our interviewers. It could also be due to the fact that opium use is quite common in Iran. So, although stigmatized, it is not quite as big a taboo as in some other countries. Fortunately, the sensitivity of reporting was similar in disease-controls and visitor-controls. This is in contrast to studies performed outside Iran that have reported substantial underreporting among controls. ${ }^{46,47}$ In addition, we found a sensitivity of $70 \%$ for self-reporting opium use among cancer patients, which was similar to underreporting of healthy visitor controls. Although in case-control studies, information bias is typically higher in the controls than in the cases and 


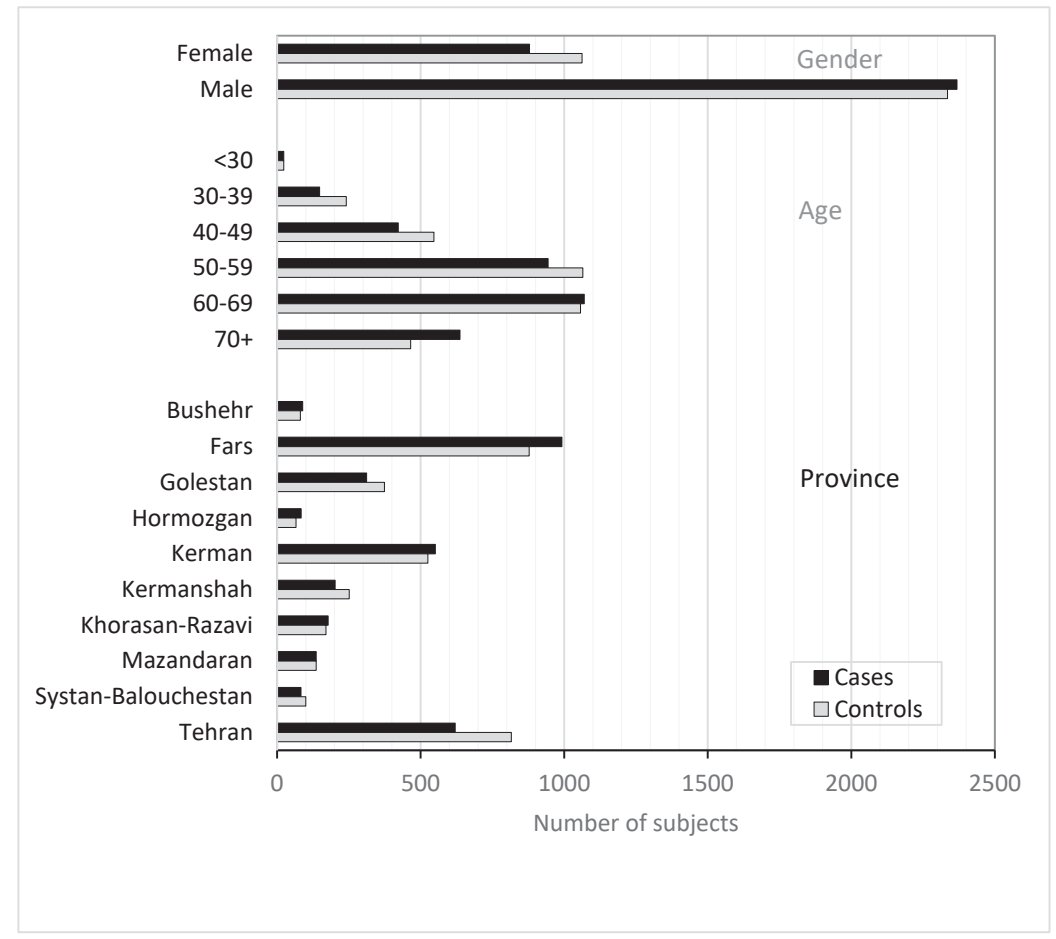

Figure 2. Numbers of Cases and Controls in the IROPICAN Study, by Gender, Age and Province.

leads to differential misclassification, here we found that underreporting for opium use was similar in the cancer patients and in the controls. Our findings suggest that we need to adjust for non-differential bias when we study the association between opium use and risk of cancers

In addition to accurate data on opium use, we have collected data on a host of other risk factors, such as various forms of tobacco use (e.g., cigarettes, water-pipe, and nass), which can be used for future epidemiologic studies. This wealth of data will also allow us to assess possible interaction effects of opium consumption and other risk factors of cancers such as water pipe and cigarette consumption, and also study the effects of opium in never tobacco users.

From each case and control, a $10 \mathrm{~mL}$ sample of venous blood was collected for DNA extraction for future epidemiological studies to evaluate the association between opium use and genetic and molecular markers of cancers - a relationship which has not been studied before. Additionally, saliva samples were collected from head and neck cases and controls to study the prevalence of HPV and detect the most prevalent type of HPV among head and neck cancer cases in Iran. The data can also be combined with opium use, tobacco use, and other data for various epidemiologic studies.

The strengths of this case-control study are its large sample size, collection of detailed data on opium use and potential confounders, validation of the questionnaires, substantial training and supervision of the interviewers, high participation rates among cases and controls, careful selection of controls, and histologic confirmation of cancer diagnoses. Despite our best efforts, however, this study, as a case-control study, may still suffer from some biases like selection bias and some reporting bias. We did not choose a population-based control group because underreporting of opium use, as a sensitive matter, in the general population may be substantially higher than hospital visitors. ${ }^{48}$

In conclusions, we were able to successfully design and test various steps in conducting this large multi-center study. The full study with general questionnaires, food frequency questionnaires, and biological samples is by far the largest study of association on opium and cancer. The findings from this study are expected to make a significant contribution to advancing our knowledge on carcinogenicity of opium use. The study data can also be used to assess the effects of other potential risk factors of cancer, such as water-pipe smoking in Iran.

\section{Authors' Contribution}

All authors of this research paper have directly participated in the planning, execution, or analysis of the study. $M H, H R, M M$, $\mathrm{MGH}, \mathrm{EM}, \mathrm{EE}, \mathrm{AAH}, \mathrm{AR}, \mathrm{AR}, \mathrm{ARM}, \mathrm{PB}, \mathrm{EW}, \mathrm{FK}, \mathrm{KZ}, \mathrm{AM}, \mathrm{FN}, \mathrm{RS}$, $A N, M S, A A, A N, R S, A S, M B, M M, E P, H P, S E, O N, S S, R S, A N$ contributed to the design and implementation of the study. KZ, FK, $\mathrm{EP}$, as corresponding authors supervised the conduct and analyses of this study. All authors of this paper have read and approved the final version submitted. The contents of this manuscript have not been copyrighted or published previously. The contents of this manuscript are not now under consideration for publication elsewhere. The contents of this manuscript will not be copyrighted, submitted, or published elsewhere while acceptance by the journal is under consideration. There are no directly related manuscripts or abstracts, published or unpublished, by any authors of this paper. 
Conflict of Interest Disclosures

None declared.

\section{Disclaimer}

Where authors are identified as personnel of the International Agency for Research on Cancer/World Health Organization, the authors alone are responsible for the views expressed in this article and they do not necessarily represent the decisions, policy or views of the International Agency for Research on Cancer/World Health Organization.

\section{Ethical Statement}

The study was approved by the Ethics Committee of the National Institute of Medical Research Development (NIMAD) (Code: IR.NIMAD.REC.1394.027). All participants signed written inform consents, and data were handled confidentially.

\section{Acknowledgements}

The IROPICAN study was funded by the National Institute for Medical Research Development (NIMAD) (grant number: 940045). In addition, this paper uses elements of the theses of two PhD students, Maryam Marzban funded by Fars University of Medical Sciences (grant number: 94 7650), and Hamideh Rashidian funded by Kerman University of Medical Sciences (grant number: 9421). Also, we would like to thank the Clinical Research Development Center of the Persian Gulf Martyrs Hospital affiliated to Bushehr University of Medical Sciences and all other hospitals in different provinces hosting the project, which facilitated the study.

\section{References}

1. World drug report 2019 (United Nations publication, sales no. E.19.XI.8).

2. Amin-Esmaeili M, Rahimi-Movaghar A, Sharifi V, Hajebi A, Radgoodarzi R, Mojtabai R, et al. Epidemiology of illicit drug use disorders in Iran: prevalence, correlates, comorbidity and service utilization results from the Iranian Mental Health Survey. Addiction. 2016;111(10):1836-47. doi: 10.1111/ add.13453.

3. Nalini M, Shakeri R, Poustchi H, Pourshams A, Etemadi A Islami F, et al. Long-term opiate use and risk of cardiovascular mortality: Results from the Golestan Cohort Study. Eur J Prev Cardiol. 2020; zwaa006. doi: 10.1093/eurjpc/zwaa006.

4. Alizadeh H, Naghibzadeh-Tahami A, Khanjani N, et al. Opium use and head and neck cancers: A matched case-control study in Iran. Asian Pac J Cancer Prev. 2020;21(3):783-790. doi: 10.31557/APJCP.2020.21.3.783.

5. Mousavi MRA, Damghani MA, Haghdoust AA, Khamesipour A. Opium and risk of laryngeal cancer. Laryngoscope. 2003;113(11):1939-43. doi: 10.1097/00005537-20031100000016.

6. Mohebbi E, Hadji M, Rashidian H, Rezaianzadeh A, Marzban M, Haghdoost AA, et al. Opium use and the risk of head and neck squamous cell carcinoma. Int J Cancer. 2021;148(5):1066-1076. doi: 10.1002/ijc.33289.

7. Khoo R. Radiotherapy of carcinoma of the larynx. Ann Acad Med Singap. 1981;10(3):307-310.

8. Akbari M, Naghibzadeh TA, Khanjani N, et al. Opium as a risk factor for bladder cancer: A population-based case-control study in Iran. Arc Iran Med. 2015;18(9):567-571.

9. Afshari M, Janbabaei G, Bahrami MA, Moosazadeh M. Opium and bladder cancer: A systematic review and meta-analysis of the odds ratios for opium use and the risk of bladder cancer. PLoS One. 2017;12(6):e0178527. doi: 10.1371/journal. pone.0178527.

10. Sheikh M, Shakeri R, Poustchi H, Pourshams A, Etemadi A, Islami F, et al. Opium use and subsequent incidence of cancer: Results from the Golestan Cohort Study. Lancet Glob Health. 2020;8(5):e649-e660. doi: 10.1016/S2214-109X(20)30059-0.
11. MacLennan R, Da Costa J, Day NE, Law CH, Ng YK, Shanmugaratnam K. Risk factors for lung cancer in Singapore Chinese, a population with high female incidence rates. Int J Cancer. 1977;20(6):854-60. doi: 10.1002/ijc.2910200606.

12. Masjedi MR, Naghan PA, Taslimi S, Yousefifard M, Ebrahimi SM, Khosravi A, et al. Opium could be considered an independent risk factor for lung cancer: a case-control study. Respiration. 2013;85(2):112-8. doi: 10.1159/000338559.

13. Fahmy MS, Sadeghi A, Behmard S. Epidemiologic study of oral cancer in Fars province, Iran. Community Dent Oral Epidemiol. 1983;11(1):50-8. doi: 10.1111/j.1600-0528.1983. tb01354.x.

14. Bakhshaee M, Raziee HR, Afshari R, Amali A, Roopoosh $M$, Lotfizadeh A. Opium addiction and risk of laryngeal and esophageal carcinoma. Iran J Otorhinolaryngol. 2017;29(90):19-22.

15. Shakeri R, Malekzadeh R, Etemadi A, Nasrollahzadeh D, Aghcheli K, Sotoudeh M, et al. Opium: An emerging risk factor for gastric adenocarcinoma. Int J Cancer. 2013;133(2):455-61. doi: 10.1002/ijc.28018. Epub 2013 Feb 13.

16. Naghibzadeh TA, Khanjani N, Yazdi FV, Varzandeh $M$, Haghdoost AA. Opium as a risk factor for upper gastrointestinal cancers: A population-based case-control study in Iran. Arc Iran Med. 2014;17(1):2-6.

17. Malekzadeh MM, Khademi $\mathrm{H}$, Pourshams A, Etemadi A, Poustchi $\mathrm{H}$, Bagheri $\mathrm{M}$, et al. Opium use and risk of mortality from digestive diseases: A prospective cohort study. Am J Gastroenterol. 2013;108(11):1757-65. doi: 10.1038/ ajg.2013.336.

18. Ghadirian P, Stein GF, Gorodetzky C, Roberfroid MB, Mahon $\mathrm{GA}$, Bartsch $\mathrm{H}$, et al. Oesophageal cancer studies in the Caspian Littoral of Iran: Some residual results, including opium use as a risk factor. Int J Cancer. 1985;35(5):593-7. doi: 10.1002/ijc.2910350505.

19. Shakeri R, Kamangar F, Nasrollahzadeh D, Nouraie M, Khademi $\mathrm{H}$, Etemadi $\mathrm{A}$, et al. Is opium a real risk factor for esophageal cancer or just a methodological artifact? Hospital and neighborhood controls in case-control studies. PLoS One. 2012;7(3):e32711. doi: 10.1371/journal.pone.0032711.

20. Nasrollahzadeh D, Kamangar F, Aghcheli K, Sotoudeh M, Islami F, Abnet CC, et al. Opium, tobacco, and alcohol use in relation to oesophageal squamous cell carcinoma in a high-risk area of Iran. Br J Cancer. 2008;98(11):1857-63. doi: 10.1038/sj.bjc.6604369.

21. Moossavi S, Mohamadnejad M, Pourshams A, Poustchi H, Islami F, Sharafkhah M, et al. Opium use and risk of pancreatic cancer: A prospective cohort study. Cancer Epidemiol Biomarkers Prev. 2018;27(3):268-273. doi: 10.1158/10559965.EPI-17-0592.

22. Shakeri R, Kamangar F, Mohamadnejad M, Tabrizi R, Zamani F, Mohamadkhani A, et al. Opium use, cigarette smoking, and alcohol consumption in relation to pancreatic cancer. Medicine (Baltimore). 2016;95(28):e3922. doi: 10.1097/ MD.0000000000003922.

23. Lankarani KB, Khosravizadegan Z, Naghibzadeh-Tahami A, Akbari M, Khodadost M, Honarvar B, et al. Opium use and risk of lower gastrointestinal cancers: Population-based casecontrol study in south of Iran. Int J Cancer Manag. 2017;10(6): e8227.

24. Naghibzadeh-Tahami A, Yazdi Feyzabadi V, Khanjani N, Ashrafi-Asgarabad A, Alizaeh H, Borhaninejad VR, et al. Can opium use contribute to a higher risk of colorectal cancers? A matched case-control study in Iran. Iran J Public Health. 2016;45(10):1322-1331.

25. IARC Monographs Vol 126 group. Carcinogenicity of opium consumption. Lancet Oncol. 2020;21(11):1407-1408. doi: 10.1016/S1470-2045(20)30611-2

26. Sheikh M, Kamangar F, Malekzadeh R. Fifty years of research 
and one conclusion: Opium causes cancer. Arch Iran Med. 2020;23(11):757-760. doi: 10.34172/aim.2020.95.

27. Kamangar F, Shakeri R, Malekzadeh R, Islami F. Opium use: An emerging risk factor for cancer? Lancet Oncol. 2014;15(2):e69-77. doi: 10.1016/S1470-2045(13)70550-3

28. Estimation of the population size of substance and alcohol abusers in Iran. Research Center for Modeling in Health, Institute for Futures Studies in Health, Biostatistics and Epidemiology Department, Health School, Kerman University of Medical Sciences, Kerman, Iran; 2012:43-45.

29. Pourshams A, Khademi H, Malekshah AF, Islami F, Nouraei M, Sadjadi AR, et al. Cohort profile: The Golestan Cohort Study a prospective study of oesophageal cancer in northern Iran. Int J Epidemiol. 2010;39(1):52-9. doi: 10.1093/ije/dyp161.

30. Vasheghani-Farahani A, Tahmasbi $M$, Asheri $H$, Ashraf $H$, Nedjat S, Kordi R. The Persian, last 7-day, long form of the international physical activity questionnaire: Translation and validation study. Asian J Sports Med. 2011;2(2):106-16. doi: 10.5812/asjsm.34781

31. Poustchi H, Eghtesad S, Kamangar F, Etemadi A, Keshtkar AA, Hekmatdoost A, et al. Prospective epidemiological research studies in Iran (the PERSIAN Cohort Study): Rationale, objectives, and design. Am J Epidemiol. 2018;187(4):647655. doi: 10.1093/aje/kwx314.

32. Centers for Disease Control and Prevention. National health and nutrition examination survey: Anthropometry procedures manual. Atlanta, GA, USA: CDC; 2007.

33. Lee DH, Keum N, Hu FB, Orav EJ, Rimm EB, Sun Q, et al. Development and validation of anthropometric prediction equations for lean body mass, fat mass and percent fat in adults using the national health and nutrition examination survey (NHANES) 1999-2006. Br J Nutr. 2017;118(10):858866. doi: 10.1017/S0007114517002665.

34. Aghaee-Afshar M, Khazaeli P, Behnam B, Rezazadehkermani M, Ashraf-Ganjooei N. Presence of lead in opium. Arch Iran Med. 2008;11(5):553-554.

35. Abnet CC, Saadatian-Elahi M, Pourshams A, Boffetta $P$, Feizzadeh A, Brennan P, et al. Reliability and validity of opiate use self-report in a population at high risk for esophageal cancer in Golestan, Iran. Cancer Epidemiol Biomarkers Prev. 2004;13(6):1068-70.

36. Rashidian H, Hadji M, Marzban M, Gholipour M, RahimiMovaghar A, Kamangar F, et al. Sensitivity of self-reported opioid use in case-control studies: Healthy individuals versus hospitalized patients. PLoS One. 2017;12(8):e0183017. doi: 10.1371/journal.pone.0183017.

37. Mohebbi E, Kamangar F, Rahimi-Movaghar A, Haghdoost AA, Etemadi A, Amirzadeh S, et al. An exploratory study of units of reporting opium use in Iran: Implications for epidemiologic studies. Arc Iran Med. 2019;22(10):541-545.

38. Nikfarjam A, Shokoohi M, Shahesmaeili A, Haghdoost AA, Baneshi MR, Haji-Maghsoudi S, et al. National population size estimation of illicit drug users through the network scaleup method in 2013 in Iran. Int J Drug Policy. 2016;31:147-52. doi: 10.1016/j.drugpo.2016.01.013.

39. Nakhaee N, Divsalar K, Meimandi MS, Dabiri S. Estimating the prevalence of opiates use by unlinked anonymous urine drug testing: A pilot study in Iran. Subst Use Misuse. 2008;43(34):513-20. doi: 10.1080/10826080701772348.

40. Ziaaddini $\mathrm{H}$, Ziaaddini MR. The household survey of drug abuse in Kerman, Iran. J Applied Sci. 2005;5(2):380-382.

41. Bernaards CM, Twisk JW, Snel J, Van Mechelen W, Kemper HC. Is calculating pack-years retrospectively a valid method to estimate life-time tobacco smoking? A comparison between prospectively calculated pack-years and retrospectively calculated pack-years. Addiction. 2001;96(11):1653-61. doi: 10.1046/j.1360-0443.2001.9611165311.x.

42. Islami F, Kamangar F, Nasrollahzadeh D, Aghcheli K, Sotoudeh M, Abedi-Ardekani B, et al. Socio-economic status and oesophageal cancer: Results from a populationbased case-control study in a high-risk area. Int J Epidemiol. 2009;38(4):978-88. doi: 10.1093/ije/dyp195

43. Caraceni A, Hanks G, Kaasa S, Bennett MI, Brunelli C, Cherny $\mathrm{N}$, et al. Use of opioid analgesics in the treatment of cancer pain: Evidence-based recommendations from the EAPC. Lancet Oncol. 2012;13(2):e58-68. doi: 10.1016/\$14702045(12)70040-2.

44. Zeppetella G, Davies AN. Opioids for the management of breakthrough pain in cancer patients. Cochrane Database Syst Rev. 2013;(10):CD004311. doi: 10.1002/14651858. CD004311.pub3.

45. Wang W. Illegal drug abuse and the community camp strategy in China. J Drug Educ. 1999;29(2):97-114. doi: 10.2190/J28RFH8R-68A9-L288.

46. Shuster JJ, Cook B. Hospital or population controls: A discussion. J Chronic Dis. 1983;36(4):315-6. doi: 10.1016/0021-9681(83)90116-9.

47. Stavraky KM, Clarke EA. Hospital or population controls? An unanswered question. J Chronic Dis. 1983;36(4):301-7. doi: 10.1016/0021-9681(83)90113-3.

48. Mohebbi E, Rashidian H, Naghibzadeh Tahami A, Haghdoost AA, Rahimi-Movaghar A, Seyyedsalehi MS, et al. Opium use reporting error in case-control studies: neighborhood controls versus hospital visitor controls. Med J Islam Repub Iran. 2021;35(1):457-63. 